\title{
基于弹尾虫运动机制的平衡轮式跳跃机器人 的设计
}

\author{
陈子明 1,2 卢杰 ${ }^{1,2}$ 邓朋 ${ }^{1,2}$ 郭 玉 ${ }^{1,2}$ 李艳文 ${ }^{1,2}$ \\ (1. 燕山大学河北省并联机器人与机电系统实验室 秦皇岛 066004; \\ 2. 燕山大学机械工程学院 秦皇岛 066004)
}

\begin{abstract}
摘要: 针对弹尾虫的生物体结构与运动特性, 复合轮式与跳跃运动设计了一种新型跳跃机器人。利用弹尾虫独特的跳跃运动机制 设计了跳跃原理结构, 并使用拉格朗日法完成跳跃原理结构着地起跳、滕空阶段动力学方程的构建与仿真, 分析并验证了跳跃过 程及跳跃原理的可行性。通过轮式结构的复合设计, 使得机器人具备平衡轮式移动特性, 展现跳跃原理结构的可移植性。搭建机 器人控制系统, 使用 3D 打印技术完成样机制作, 最终设计机器人长 $15 \mathrm{~cm}$ 、宽 $9.5 \mathrm{~cm}$ 、高 $7.5 \mathrm{~cm}$, 整体重 $268.6 \mathrm{~g}$ 。试验测试表 明, 机器人具备快速连续运动性能, $1 \mathrm{~s}$ 内完成能量蓄积与释放, $0.5 \mathrm{~s}$ 内实现姿态复位; 具备多运动模式, 可实现高 $20 \mathrm{~cm}$ 、远 $15 \mathrm{~cm}$ 的跳跃动作, 同时可完成跳跃后的姿态复位, 平衡轮式移动以及传统平衡小车难以实现的倒地后自主平衡复位。
\end{abstract}

关键词: 弹尾虫; 跳跃机器人; 动力学; 平衡轮; 自复位

中图分类号: TP242

\section{Design of Balanced Wheeled Jumping Robot Based on the Motion Mechanism of Springtail}

\author{
CHEN Ziming ${ }^{1,2}$ LU Jie $^{1,2}$ DENG Peng ${ }^{1,2}$ GUO Yu ${ }^{1,2}$ LI Yanwen ${ }^{1,2}$
}

(1. Hebei Provincial Key Laboratory of Parallel Robot and Mechatronic System, Yanshan University,

Qinhuangdao 066004;

2. School of Mechanical Engineering, Yanshan University, Qinhuangdao 066004)

\begin{abstract}
A new type of jumping robot is designed according to the biological structure and motion characteristics of the springtail, combined with wheel and jumping motion. The principlem echanism of jumping is designed by imitating the unique jumping mechanism of the springtail, and the dynamics equation of jumping mechanism in take-off and flight phase are built by Lagrange method and the model is simulated to verify the feasibility of the jumping principle. Through the composite design of wheel structure, the robot has the characteristics of balanced wheel movement, which reflects the portability of jumping mechanism. The robot control system was built, and the prototype was made by using 3D printing technology. The overall length, width, height and weight of the system were $15 \mathrm{~cm}, 9.5 \mathrm{~cm}, 7.5 \mathrm{~cm}$ and $268.6 \mathrm{~g}$ respectively. Experiments show that the robot has fast and continuous motion performance, energy storage and release can be completed in $1 \mathrm{~s}$, and posture reset can be achieved in $1.5 \mathrm{~s}$. With multi-motion mode, it can achieve the jump action of $20 \mathrm{~cm}$ high and $15 \mathrm{~cm}$ far, at the same time, it can complete the posture reset after jumping, balance wheeled movement, and self-balancing reset after falling down which is difficult to achieve by traditional balance car.
\end{abstract}

Key words: springtail; jumping robot; dynamics; balancing wheel; self-righting

\section{0 前言}

跳跃是自然界中体型较小生物常用的越障方 式。与其他移动方式相比, 由于跳跃运动着地点的

* 国家自然科学基金(51775474)和河北省重点研发计划(19221909D)资助 项目。20191011 收到初稿, 20200115 收到修改稿
离散性和发力的爆发性, 使得生物体在高于身体数 倍的障碍物前具有其他运动方式无法比拟的越障优 势 ${ }^{[1]}$ 。模仿自然生物的跳跃运动形式增强了移动机 器人机动性能, 弥补了传统机器人移动性能的不足。 因此跳跃运动的仿生设计对提高机器人的越障能力 具有重要意义。

弹尾虫是自然界中一类独特的跳跃生物, 具备 单独作用的跳跃器官。独特的跳跃运动机制使得弹 
尾虫具有超高跳跃能力的同时具备了复合结构特 性。这种兼容性能良好的复合设计为传统轮式移动 机器人突破常规道路限制提供了新的思路, 同时也 为单一跳跃机器人移动效率的不足进行了弥补。国 外对仿生跳跃机器人的研究较早并取得了较好的成 果, 如 VALENTIN 等 ${ }^{[2]}$ 设计了一种能够跳跃 $3.35 \mathrm{~m}$ 高的仿蝗虫跳跃机器人, 首尔国立大学 JUNG 等 ${ }^{[3]}$ 依据沫禅的跳跃形式设计了一种依靠线性弹簧与记 忆合金驱动的微型跳跃机器人, 加州大学伯克利分 校使用扭簧与连杆研发了一款最大跳跃高度能达到 $1 \mathrm{~m}$ 的仿贞猴机器人 Salto $^{[4]}$, 以及麻省理工学院设 计的可连续跳跃的 JelloCube ${ }^{[5]}$ 等。国内也展开了相 应研究并取得了一定的研究成果, 如北京航空航天 大学的陈殿生等 ${ }^{[6]}$ 设计了一种可实现跳跃与爬行的 仿生机器人, 东南大学的 ZHANG 等 ${ }^{[7]}$ 设计了一种 可倒地翻转的仿蝗虫跳跃机器人, 北京邮电大学的 张小飞等 ${ }^{[8]}$ 设计了一种可跳跃式两轮机器人, 西北 工业大学的鲍延年等 ${ }^{[9]}$ 设计了一种通过滑翔运动提高 跳跃性能的折叠翼弹跳机器人。国内外侧重于对跳跃 机器人单一跳跃性能的研究, 存在运动形式单一、复 合运动结构复杂, 跳跃结构和其他运动结构存在结构 与运动控制上的干涉等问题, 同时在跳跃机构执行效 率、多模式运动及运动连续性上展现出较大的不足。 弹尾虫独特的运动机制体现了多运动模式的优 势、在良好跳跃性能的基础上具备结构简单执行效 率高等特点, 为设计复合跳跃机器人提供了新的设 计思路。因此, 本文通过对弹尾虫运动机制的研究, 结合平面连杆与变胞运动副的特点提出一种新型跳 跃机构。在兼容传统平衡轮式移动结构的基础上形 成一种多运动模式的跳跃机器人, 具备跳跃与轮式 移动的同时也可实现跳跃后的姿态复位以及平衡自 复位功能。

\section{1 原理结构设计}

\section{1 弹尾虫结构}

弹尾虫 ${ }^{[10]}$ 也叫跳虫, 体长一般仅有 $1 \sim 5 \mathrm{~mm}$, 能依赖在腹部末端的弹跳器官进行强而有力的跳 跃。当遇到敌害时, 由于肌肉的伸张, 弹跳器官猛 向下后方弹击, 使身体向前一跃, 一次可跳出几乎 是它身体的 200 倍的距离, 跳跃能力不输于跳虫。 如图 1 所示, 弹尾虫身体结构主要由头、胸和腹部 三部分组成。弹跳器官与腹部末端相连, 一般状态 下收拢于腹部的腹沟中。

\section{2 弹尾虫运动机制}

与常见跳跃物种蝗虫、跳蚤、沫蝉等不同, 具

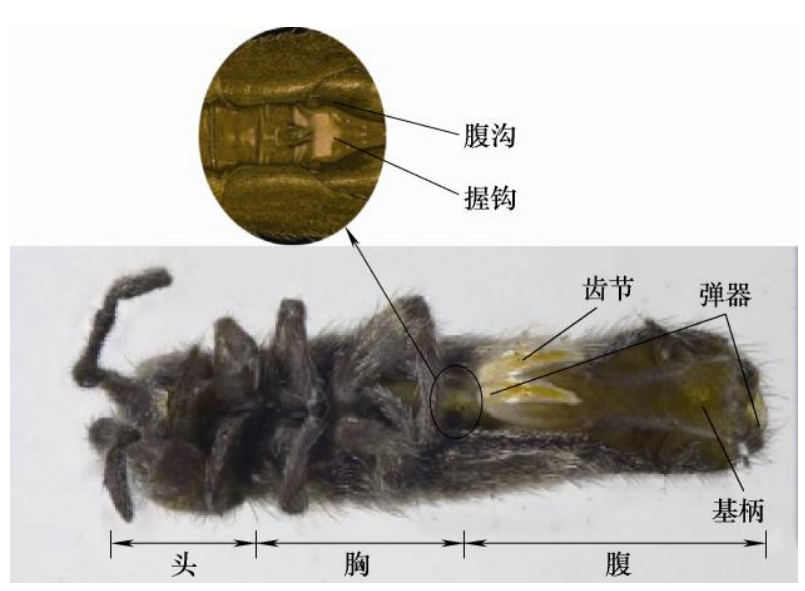

图 1 弹尾虫身体结构

备单独跳跃器官的弹尾虫避免了耦合式结构的干涉 从而收获了更加优越与高效的跳跃性能。如图 1 所 示, 弹尾虫的跳跃器官叫做弹器, 主要由基柄与齿 节两部分组成。这种弹跳器官常折叠于身体腹部下 的腹沟中, 且夹持在握钩上 ${ }^{[11]}$ 。跳跃时弹尾虫腹部 的握钩会松开, 取消对弹器的握持, 进而基柄绕着 腹部末端转动。在齿节与地面进行接触完成快速的 相互作用后, 弹尾虫产生跳跃运动的所需的加速度, 如图 2 所示 ${ }^{[12]}$ 。

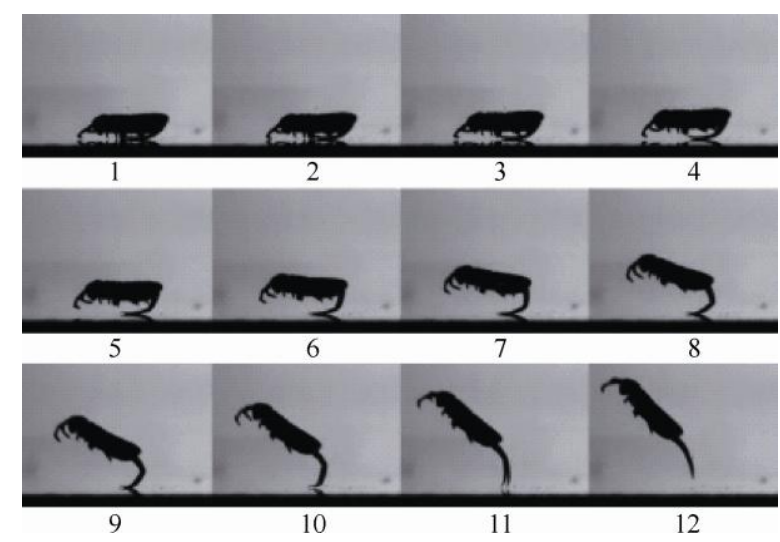

图 2 弹尾虫跳跃过程

弹尾虫兼具爬行与跳跃能力, 在结构与运动上 具备良好的兼容性。腹部的握钩能够将弹跳器官钩 持在腹部下方, 避免弹器绕腹部末端的旋转运动。 腹沟的作用类似箱体, 弹器被包裹在腹沟中避免了 与爬行结构干涉的同时也对实现了对弹器的保护 作用。

\section{3 跳跃原理结构设计}

基于弹尾虫的这种兼容性良好的跳跃运动机 制, 结合平面连杆机构与变胞运动副的优势实现跳 跃原理结构的设计, 如图 3 所示。

整体结构与弹尾虫跳跃结构一致，包含了基柄 杆、齿节杆、扭簧、机身与弧形滑道等。基柄杆的 一端为旋转驱动副, 通过旋转运动实现跳跃运动驱 


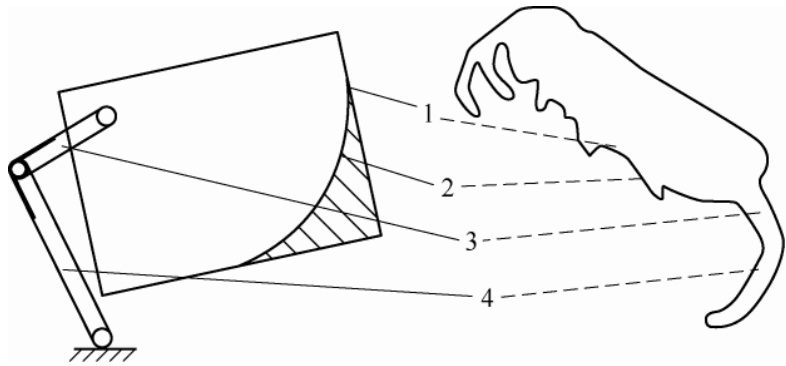

图 3 跳跃原理结构

1. 机身 2. 滑道 3. 基柄杆 4. 齿节杆

动。齿节杆为与地面相互作用的连杆, 提供跳跃的 动力传递。基柄杆与齿节杆由蓄能元件扭簧连接, 形成的变胞运动副可作为蓄积能量的储能部分, 也 可在驱动反向旋转时使基柄与齿节杆成为一体。机 身内部为空腔与弹尾虫腹沟作用类似, 保证跳跃运 动同时可对跳跃结构起到保护作用。滑道起到了握 钩的作用, 实现跳跃的蓄能与释放的功能。具体的 跳跃过程如图 4 所示。

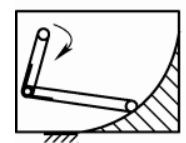

(a) 蓄能段

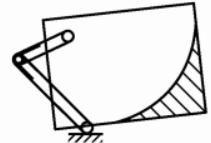

(b) 起跳段

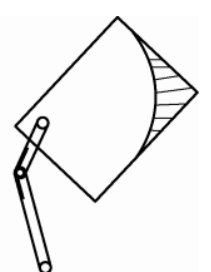

(c) 腾空段
图 4 跳跃过程

由扭簧构成的变胞运动副为跳跃后姿态复位提 供了作用机制。如图 5 所示, 非蓄能状态时, 由于 扭簧反向刚度较大, 驱动反向旋转会使得基柄与齿 节杆整体反向旋转, 从而实现了姿态复位。

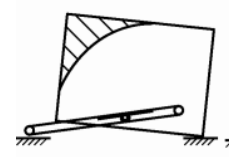

(a) 落地翻转姿态

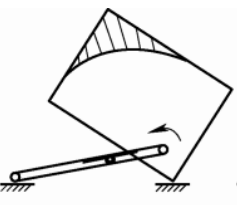

(b) 机身翻转

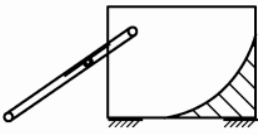

(c) 姿态转正
图 5 姿态复位

\section{2 动力学模型构建}

\section{1 蓄能分析}

跳跃机器人蓄能阶段是机器人将电能转化为扭 簧的弹性势能的过程, 此阶段所蓄积的能量是跳跃 过程的基础, 也决定了最大的跳跃限度。建立如图 6 所示的几何模型, 通过分析此过程可明确蓄能过程 电动机输入转矩的变化, 以及最大弹性势能的计算。

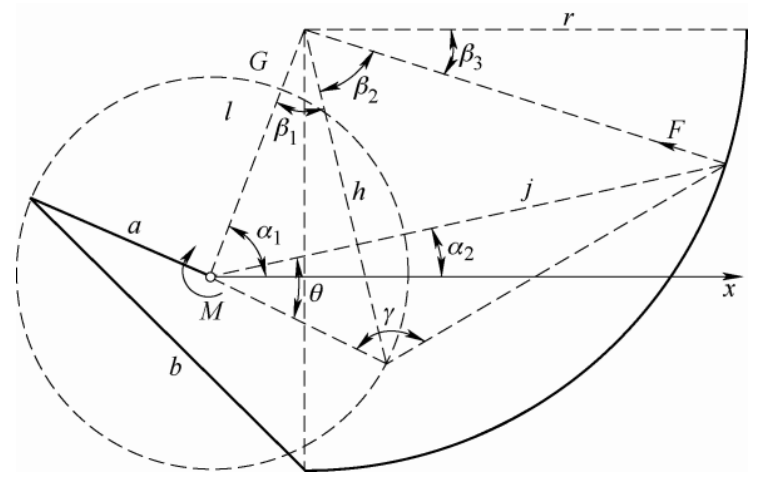

图 6 蓄能模型

图 6 中 $M$ 表示电动机的输入扭矩, $M$ 的计算公 式如式(1)所示

$$
M=F \cdot \cos \beta_{3} \cdot l \cdot \sin \alpha_{1}+F \cdot \sin \beta_{3} \cdot l \cdot \cos \alpha_{1}
$$

式中, $F=\frac{k \cdot(\pi-\gamma)}{b \cdot \cos \left(\theta-\alpha_{2}+\gamma-\beta_{3}-\pi / 2\right)}, k$ 为扭簧的 刚度， $a$ 表示基柄杆长， $b$ 表示齿节杆长; $l$ 表示弧 形滑道曲率中心 $G$ 到基柄杆转动中心的距离。

图 6 中满足的几何条件如式(2) (4)所示

$$
\begin{gathered}
h^{2}=l^{2}+a^{2}-2 l \cdot a \cdot \cos \left(\alpha_{1}+\theta-\alpha_{2}\right) \\
\frac{r}{\sin \left(\alpha_{1}-\alpha_{2}\right)}=\frac{a+b}{\sin \left(\pi-\alpha_{1}\right)} \\
j^{2}=l^{2}+r^{2}-2 l \cdot r \cdot \cos \left(\beta_{1}+\beta_{2}\right)
\end{gathered}
$$

\section{2 跳跃建模}

建立如图 7 所示的坐标系，利用质心坐标方程 得出各杆件质心的位置坐标。

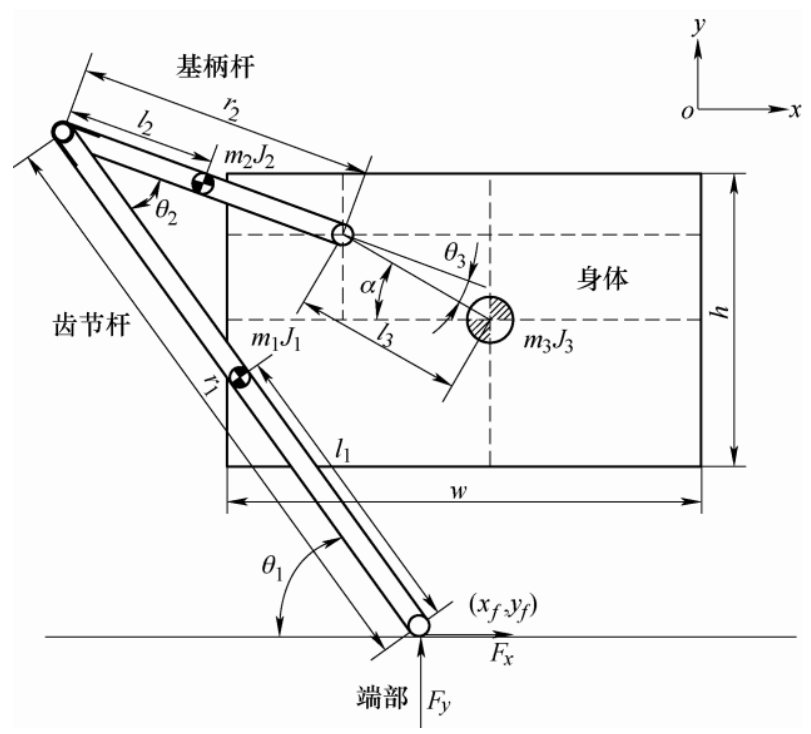

图 7 弹跳运动模型

图 7 中以齿节杆的端部作为机器人局部坐标系 的起点, 局部坐标系初始时可设置为 $(0,0)$ 。 $\theta_{1}$ 为齿 节杆与水平地面间的夹角， $\theta_{2}$ 为齿节杆与基柄杆间 
的夹角, $l_{1} 、 l_{2} 、 l_{3}$ 分别表示三部分构件质心相对于 构件一端的距离, $r_{1} 、 r_{2}$ 表示齿节与基柄的杆长, $w$ 与 $h$ 分别表示身体部分的宽与高。 $O$ 点为全局坐标 系原点, $x_{f} 、 y_{f}$ 表示齿节端点在全局坐标系中的位置 坐标, $F_{x} 、 F_{y}$ 表示齿节端点与地面在水平与坚直方 向上的受力, $J_{1} 、 J_{2} 、 J_{3}$ 表示三部分构件绕质心转动 的转动惯量。 $\alpha$ 表示基柄端点和身体质心连线与身 体长度方向的夹角。

根据图 7 所示坐标系建立各杆件的质心坐标, 方程如式(5)所示

$$
\left\{\begin{array}{l}
x_{1}=x_{f}-l_{1} \cos \left(\theta_{1}\right) \\
y_{1}=y_{f}+l_{1} \sin \left(\theta_{1}\right) \\
x_{2}=x_{f}-r_{1} \cos \left(\theta_{1}\right)+l_{2} \cos \left(\theta_{1}-\theta_{2}\right) \\
y_{2}=y_{f}+r_{1} \sin \left(\theta_{1}\right)-l_{2} \sin \left(\theta_{1}-\theta_{2}\right) \\
x_{3}=x_{f}-r_{1} \cos \left(\theta_{1}\right)+r_{2} \cos \left(\theta_{1}-\theta_{2}\right)+l_{3} \cos \left(\theta_{3}+\theta_{1}-\theta_{2}\right) \\
y_{3}=y_{f}+r_{1} \sin \left(\theta_{1}\right)-r_{2} \sin \left(\theta_{1}-\theta_{2}\right)-l_{3} \sin \left(\theta_{3}+\theta_{1}-\theta_{2}\right)
\end{array}\right.
$$

由式(5)可得模型整体质心的位置坐标, 如式(6) 所示

$$
\left\{\begin{array}{l}
X_{c}=\sum_{i=1}^{3} m_{i} x_{i} / \sum_{i=1}^{3} m_{i} \\
Y_{c}=\sum_{i=1}^{3} m_{i} y_{i} / \sum_{i=1}^{3} m_{i}
\end{array}\right.
$$

为简化动力学计算过程, 忽略关节转动摩擦、 扭簧阻尼与空气阻力对跳跃过程的影响。采用拉格 朗日方法进行跳跃原理结构动力学正问题的求解, 得到在弹性力的作用下质心位置变化及各关节转角 的变化。在求解过程中将跳跃过程分为着地起跳与 腾空阶段两个阶段进行分析 ${ }^{[13]}$ 。拉格朗日方法描述 如下

$$
\frac{\mathrm{d}}{\partial t} \frac{\partial T}{\partial \dot{q}_{i}}-\frac{\partial T}{\partial q_{i}}+\frac{\partial V}{\partial q_{i}}=Q_{i}
$$

式中, $T$ 为系统动能; $V$ 为系统势能; $q_{i}$ 为广义坐 标; $Q_{i}$ 为相应的广义力。

具体的能量表达式如式(8)所示

$$
\left\{\begin{array}{l}
T=\frac{1}{2} \sum_{i=1}^{3} J_{i} \omega_{i}^{2}+\frac{1}{2} \sum_{i=1}^{3} m_{i} v_{i}^{2} \\
V=\sum_{i=1}^{3} g m_{i} y_{i}+\frac{1}{2} k\left(\pi-\theta_{2}\right)^{2}
\end{array}\right.
$$

式中, $T$ 为起跳过程中的三个构件的转动动能与平 动动能之和, $V$ 为三个构件在某一位置时重力势能 与扭簧的弹性势能之和, $k$ 表示扭簧刚度。

着地起跳时, 将齿节末端与地面简化为铰链, 且将全局坐标系原点设置在铰链位置处。取 $\theta_{1} 、 \theta_{2}$
为广义坐标。根据式(9)可构建着地起跳阶段的拉格 朗日方程如下

$$
\left\{\begin{array}{l}
\frac{\mathrm{d}}{\mathrm{d} t} \frac{\partial T}{\partial \frac{\mathrm{d} \theta_{1}(t)}{\mathrm{d} t}}-\frac{\partial T}{\partial \theta_{1}(t)}+\frac{\partial V}{\partial \theta_{1}(t)}=0 \\
\frac{\mathrm{d}}{\mathrm{d} t} \frac{\partial T}{\partial \frac{\mathrm{d} \theta_{2}(t)}{\mathrm{d} t}}-\frac{\partial T}{\partial \theta_{2}(t)}+\frac{\partial V}{\partial \theta_{2}(t)}=0
\end{array}\right.
$$

在构建运动学、动力学数学模型的基础上, 为 便于更直观地分析, 设置具体的杆件参数与扭簧参 数, 使用 Matlab 自带的 ode45 求解器解算动力学方 程, 完成对跳跃模型的具体数值仿真。求解数值方 法为四阶-五阶 Runge-Kutta 算法, 具体步长设置为 ode45 求解器中的自适应步长。

具体主要参数设置如表 1 所示。参照弹尾虫齿 节与基柄的长度比例 ${ }^{[14]}$ 约为 2.5 左右以及结合实际 需求设计大小设置齿节杆 $r_{1}$ 与基柄杆 $r_{2}$ 的长度分别 为 $0.05 \mathrm{~mm} 、 0.02 \mathrm{~mm}$ 。 $l_{3}$ 表示了身体质心的位置 设置为 $0.025 \mathrm{~mm}$ 。其余参数的考虑到后续实际物理 模型的设计与制造初步设置为表 1 中数值。

表 1 主要结构参数

\begin{tabular}{cc||cc}
\hline 参数 & 数值 & 参数 & 数值 \\
\hline 质心偏角 $\alpha / \mathrm{rad}$ & 0.087 & 身体高度 $h / \mathrm{m}$ & 0.05 \\
杆 1 质量 $m_{1} / \mathrm{kg}$ & 0.01 & 杆 1 长度 $r_{1} / \mathrm{m}$ & 0.05 \\
杆 2 质量 $m_{2} / \mathrm{kg}$ & 0.01 & 杆 2 长度 $r_{2} / \mathrm{m}$ & 0.02 \\
身体 $m_{3} / \mathrm{kg}$ & 0.25 & 质心偏距 $l_{3} / \mathrm{m}$ & 0.025 \\
身体宽度 $w / \mathrm{m}$ & 0.06 & 刚度 $k /(\mathrm{N} \cdot \mathrm{m} / \mathrm{rad})$ & 0.3 \\
\hline
\end{tabular}

着地起跳阶段的初始仿真参数与初始的结构有 关, 具体设置如表 2 所示。考虑到满足斜抛运动水 平与坚直方向速度的平衡, $\theta_{1}$ 初步设置为 $\pi / 4$ 。同时 实际驱动时扭簧无法达到实际最大扭角, $\theta_{2}$ 设置小 部分残留大小为 $2 \pi / 9$, 其余初始参数为零值。

表 2 着地起跳阶段初始参数

\begin{tabular}{cc||cc}
\hline 参数 & 数值 & 参数 & 数值 \\
\hline 杆 1 角度 $\theta_{1} / \mathrm{rad}$ & $\pi / 4$ & 杆 1 角速度 $\theta_{1}^{\prime} /(\mathrm{rad} / \mathrm{s})$ & 0 \\
杆 2 角度 $\theta_{2} / \mathrm{rad}$ & $2 \pi / 9$ & 杆 2 角速度 $\theta_{2}^{\prime} /(\mathrm{rad} / \mathrm{s})$ & 0 \\
\hline
\end{tabular}

以表 2 所示参数进行着地起跳阶段的仿真。此 阶段主要完成齿节杆与地面的相互作用获得起跳所 需的着地起跳时杆件间角度 $\theta_{1} 、 \theta_{2}$ 的变化如图 8 所 示。此阶段为加速阶段, 在扭簧作用下齿节杆端部 与地面形成持续不断受力的状态。当端部坚直方向 上受力为零时完成加速阶段, 结束时间为 $0.04 \mathrm{~s}$, 对应的此阶段各部件运动轨迹如图 9 所示。

质心在水平与坚直方向上的速度、加速度变化 如图 10 与图 11 所示。 


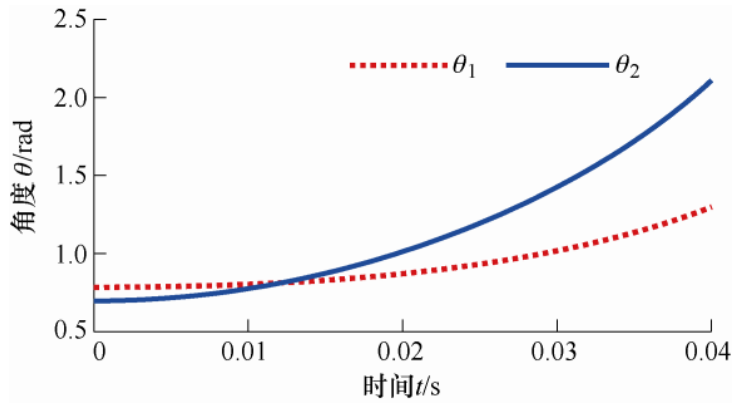

图 8 着地起跳阶段杆件角度变化

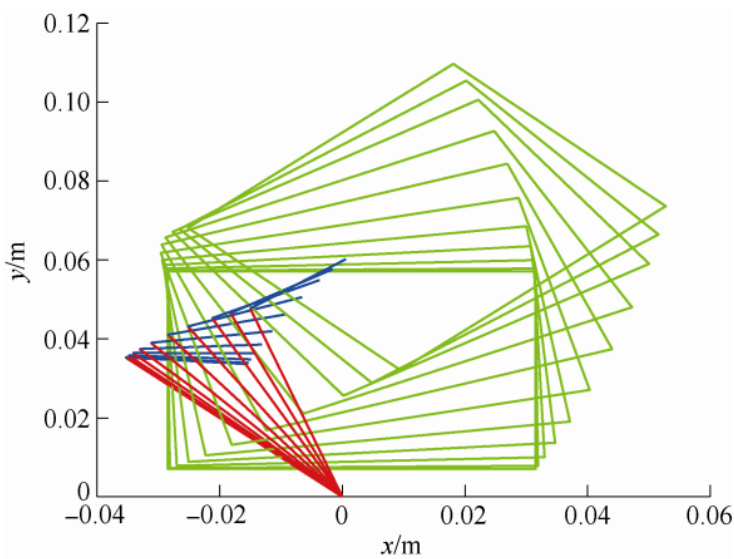

图 9 着地起跳阶段运动轨迹

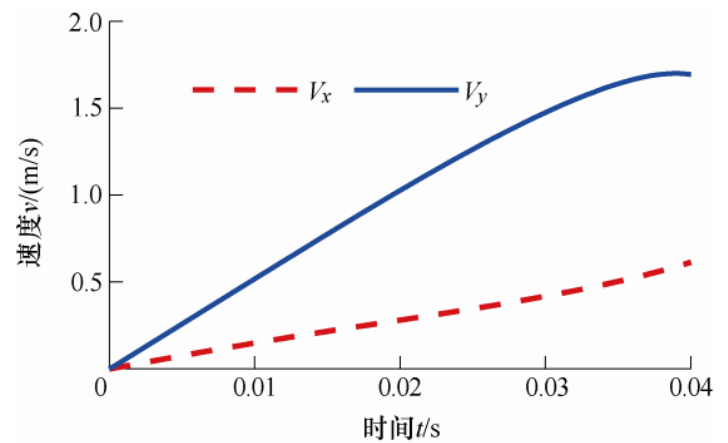

图 10 质心速度

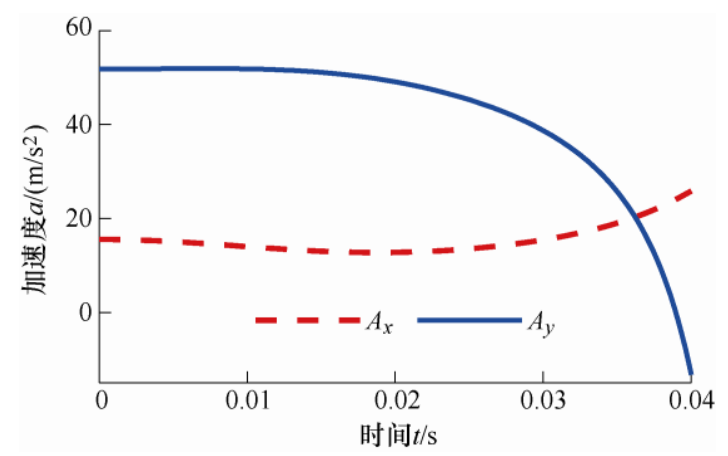

图 11 质心加速度

扭簧仅在着地起跳阶段完成对跳跃机构的加速 作用, 扭簧扭矩的变化如图 12 所示。

腾空阶段分为两部分: 腾空第一阶段与腾空第 二阶段。腾空第一阶段的特征为跳跃机构刚刚离开 地面, 齿节杆绕基柄杆旋转释放剩余弹性势能 ${ }^{[15]}$ 。 腾空第二阶段的特征为扭簧弹性势能已释放完全,

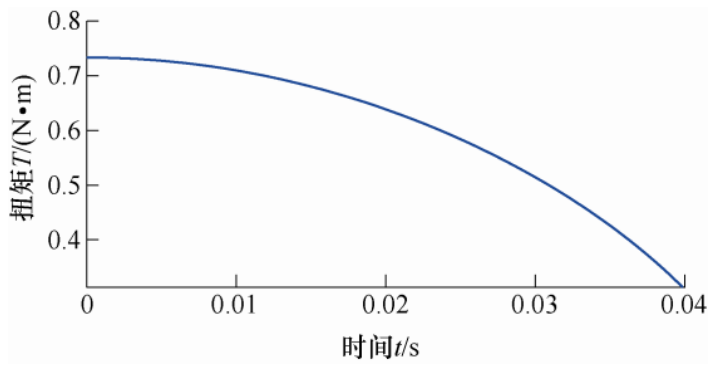

图 12 扭簧扭矩变化

齿节、基柄与身体保持固连且整体机构在空中做具 有初始速度的斜抛运动。

腾空阶段整体机构与地面无相互作用，故取消 地面与齿节端部的转动副, 以腾空前齿节杆端部为 全局坐标系原点。取 $x_{f} 、 y_{f} 、 \theta_{1} 、 \theta_{2}$ 为广义坐标，由 式(10)可构建整体在腾空阶段的拉格朗日方程

$$
\left\{\begin{array}{l}
\frac{\mathrm{d}}{\mathrm{d} t} \frac{\partial T}{\partial \frac{\mathrm{d} x_{f}(t)}{\mathrm{d} t}}-\frac{\partial T}{\partial x_{f}(t)}+\frac{\partial V}{\partial x_{f}(t)}=0 \\
\frac{\mathrm{d}}{\mathrm{d} t} \frac{\partial T}{\partial \frac{\mathrm{d} y_{f}(t)}{\mathrm{d} t}}-\frac{\partial T}{\partial y_{f}(t)}+\frac{\partial V}{\partial y_{f}(t)}=0 \\
\frac{\mathrm{d}}{\mathrm{d} t} \frac{\partial T}{\partial \frac{\mathrm{d} \theta_{1}(t)}{\mathrm{d} t}}-\frac{\partial T}{\partial \theta_{1}(t)}+\frac{\partial V}{\partial \theta_{1}(t)}=0 \\
\frac{\mathrm{d}}{\mathrm{d} t} \frac{\partial T}{\partial \frac{\mathrm{d} \theta_{2}(t)}{\mathrm{d} t}}-\frac{\partial T}{\partial \theta_{2}(t)}+\frac{\partial V}{\partial \theta_{2}(t)}=0
\end{array}\right.
$$

跳跃过程为连续过程, 腾空第一阶段的仿真初始 参数为着地起跳结束时的参数, 具体参数如表 3 所示。

表 3 腾空第一阶段初始参数

\begin{tabular}{cc||cc}
\hline 参数 & 数值 & 参数 & 数值 \\
\hline 末端水平位移 $x_{f} / \mathrm{m}$ & 0 & 末端水平速度 $x_{f}^{\prime} /(\mathrm{m} / \mathrm{s})$ & 0 \\
末端坚直位移 $y_{f} / \mathrm{m}$ & 0 & 末端坚直速度 $y_{f}^{\prime} /(\mathrm{m} / \mathrm{s})$ & 0 \\
杆 1 角度 $\theta_{1} / \mathrm{rad}$ & 1.287 & 杆 1 角速度 $\theta_{1}^{\prime} /(\mathrm{rad} / \mathrm{s})$ & 39.388 \\
杆 2 角度 $\theta_{2} / \mathrm{rad}$ & 2.113 & 杆 2 角速度 $\theta_{2}^{\prime} /(\mathrm{rad} / \mathrm{s})$ & 92.791 \\
\hline
\end{tabular}

腾空第一阶段跳跃机构离开地面，在机构跃入 空中的同时，齿节绕基柄旋转，释放残余弹性势能。 角度 $\theta_{1} 、 \theta_{2}$ 变化如图 13 所示。此阶段结束时 $\theta_{1}$ 为 $\pi$,

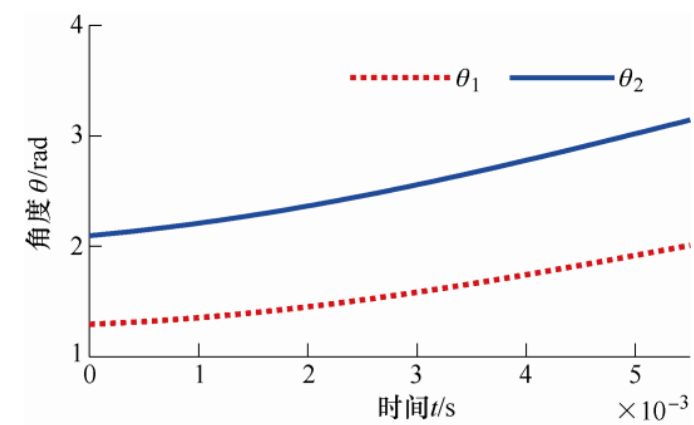

图 13 腾空第一阶段 $\theta_{1} 、 \theta_{2}$ 变化 
持续时间 $0.006 \mathrm{~s}$, 对应的轨迹运动简图如图 14 所示。

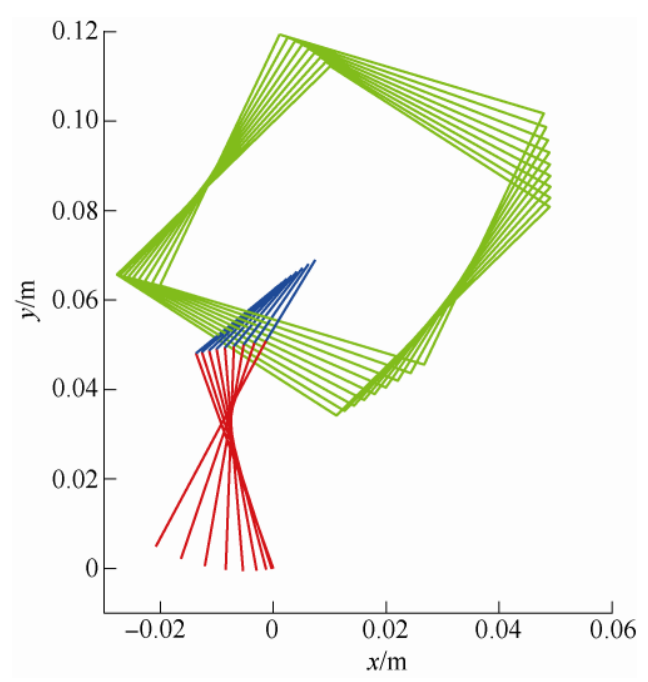

图 14 腾空第一阶段运动轨迹

同样, 腾空第一阶段计算结束时的参数为腾空 第二阶段的初始参数，仿真参数如表 4 所示。

表 4 腾空第二阶段初始参数

\begin{tabular}{cc||cc}
\hline 参数 & 数值 & 参数 & 数值 \\
\hline 末端水平位移 $x_{f} / \mathrm{m}$ & -0.021 & 末端水平速度 $x_{f}^{\prime} /(\mathrm{m} / \mathrm{s})$ & -5.398 \\
末端坚直位移 $y_{f} / \mathrm{m}$ & 0.005 & 末端坚直速度 $y_{f}^{\prime} /(\mathrm{m} / \mathrm{s})$ & 4.295 \\
杆 1 角度 $\theta_{1} / \mathrm{rad}$ & 1.983 & 杆 1 角速度 $\theta_{1}^{\prime} /(\mathrm{rad} / \mathrm{s})$ & 178.088 \\
杆 2 角度 $\theta_{2} / \mathrm{rad}$ & 3.141 & 杆 2 角速度 $\theta_{2}^{\prime} /(\mathrm{rad} / \mathrm{s})$ & 245.923 \\
\hline
\end{tabular}

在保证腾空第二阶段中三个构件为一个整体时 需将数值仿真中弹簧的刚度值 $k$ 设置为 $500 \mathrm{~N} \cdot \mathrm{m} / \mathrm{rad}$ 以避免构件间相对运动。机构整体质心变化如图 15 所示, $X_{c} 、 Y_{c}$ 表示机构整体质心的横纵坐标值。此 阶段表明仿真可完成高度 $0.225 \mathrm{~m}$ 、距离 $0.231 \mathrm{~m}$ 的跳跃动作, 对应的各部分运动轨迹如图 16 所示。

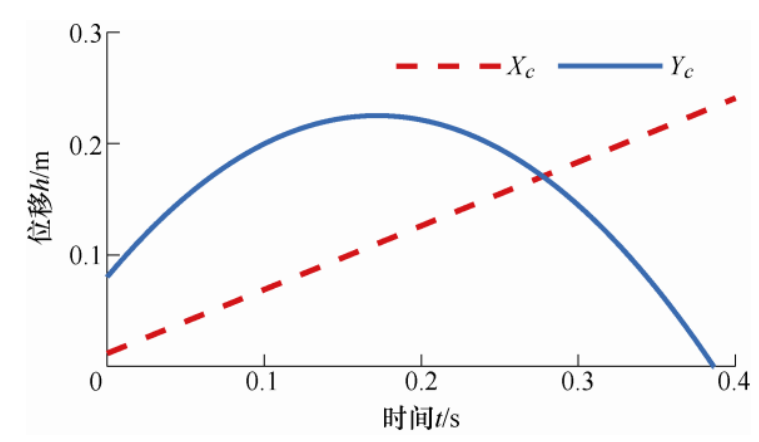

图 15 腾空第二阶段质心位移变化

\subsection{ADAMS 仿真验证}

使用 ADAMS 建立与图 7 类似的模型进行动力 学的结果验证, 确保数学模型及理论计算的准确性。

仿真后机构的质心位移如图 17 所示, 机构整体 质心坚直方向上的最大位移为 $0.218 \mathrm{~m}$, 水平方向 上的最大位移为 $0.222 \mathrm{~m}$, 结果与图 15 一致。图 18

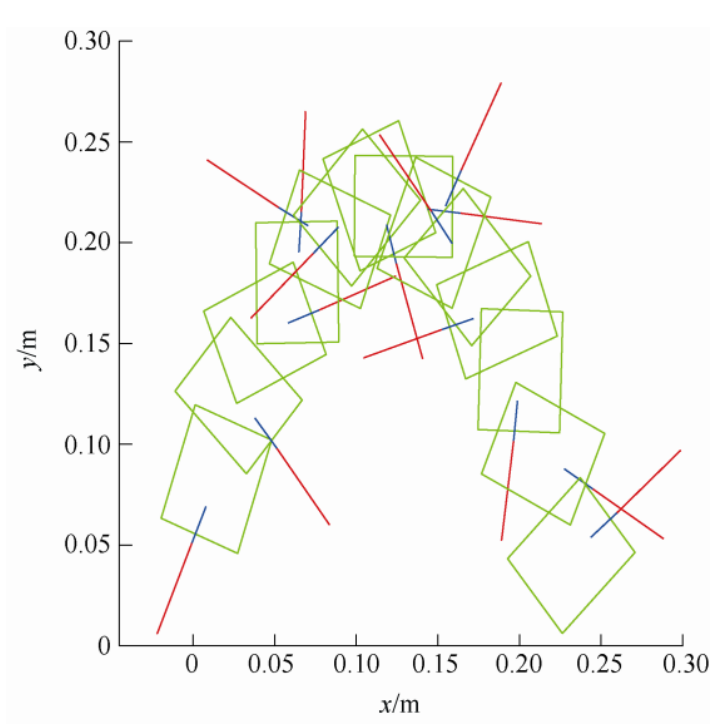

图 16 腾空第二阶段运动轨迹

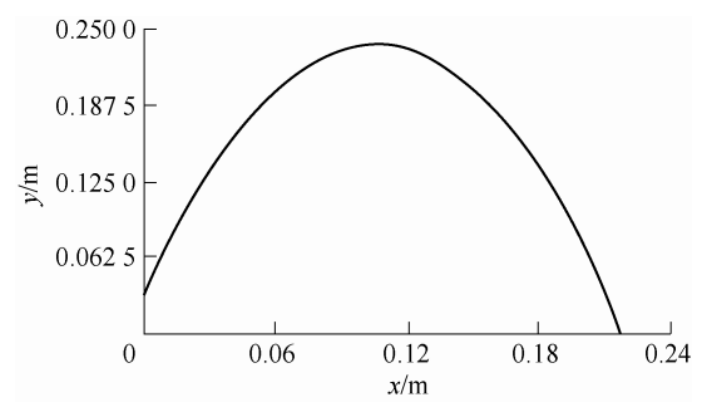

图 17 机构整体质心位移

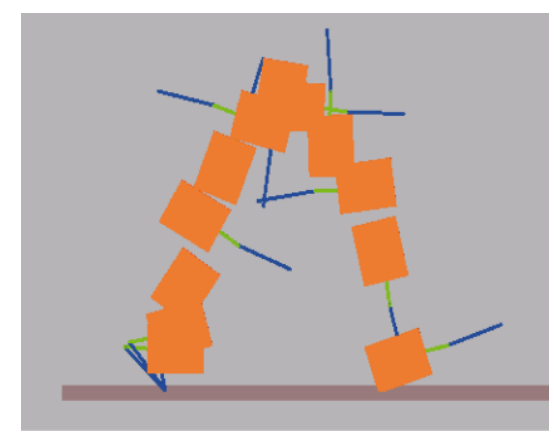

图 18 ADAMS 仿真运动轨迹

为机构跳跃过程中的姿态轨迹, 整体运动与图 16 一致。对比结果表明在拉格朗日动力学方程无法求 出解析解的情况下, 理论计算的跳跃高度与水平位 移同 ADAMS 仿真相比存在较小的求解精度误差, 且在运动姿态上相互一致, 因此可验证跳跃机构所 构建动力学模型的准确性。

\section{3 复合结构设计与功能分析}

\section{1 复合结构设计}

弹尾虫的跳跃结构具有良好的兼容性能, 在运 动结构上不会发生爬行与跳跃动作的干涉, 这为复 
合结构式的设计提供了思路。传统轮式结构具有高 效, 结构简单等特点, 因此在跳跃原理结构基础上 进行轮式结构复合, 以此来提高机器人的机动性能。 具体结构如图 19 所示。

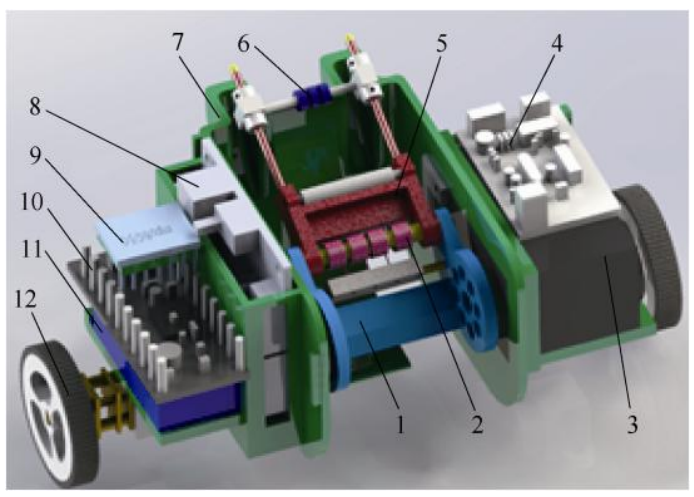

图 19 复合结构

1. 基柄结构 2. 扭簧 3. 跳跃电动机 4. 跳跃电动机驱动 5. 齿节结构 6. 滚轮 7. 机身 8. 轮式电动机驱动 9. 陀螺仪 10. 控制器 11. 电源 12. 轮式结构

\section{2 功能分析}

复合结构的设计使得跳跃机器人拥有了多种复合 运动特性, 保证了执行任务时的多机动、多灵活性能。

(1) 跳跃运动。如图 4 所示, 通过跳跃原理结 构可实现能量的蓄积与释放, 实现跳跃运动。

(2) 姿态复位。跳跃机器人在常规跳跃后可能 会出现机身倾覆的情况, 通过图 5 所示姿态复位过 程可实现姿态的矫正。

(3) 平衡轮式移动。

轮式结构的复合以及姿态传感器使得机器人能 够在传统路面上实现高效的平衡移动。

(4) 自主平衡复位

常规的平衡车无法实现自主的平衡站立, 且当 平衡倾角大于电动机提供的最大加速度对应的倾角 时便无法实现自平衡。利用变胞运动副, 在电动机 反向旋转的情况下推动机身摆正到初始平衡位置可 实现自主平衡复位，如图 20 所示。

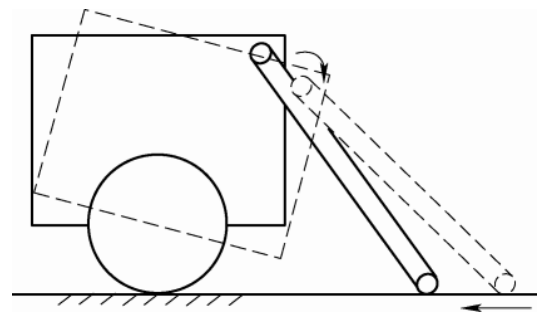

图 20 自主平衡复位

\section{4 控制系统设计}

跳跃机器人控制系统可实现运动控制、通信、
检测等功能, 具体控制系统结构如图 21 所示。

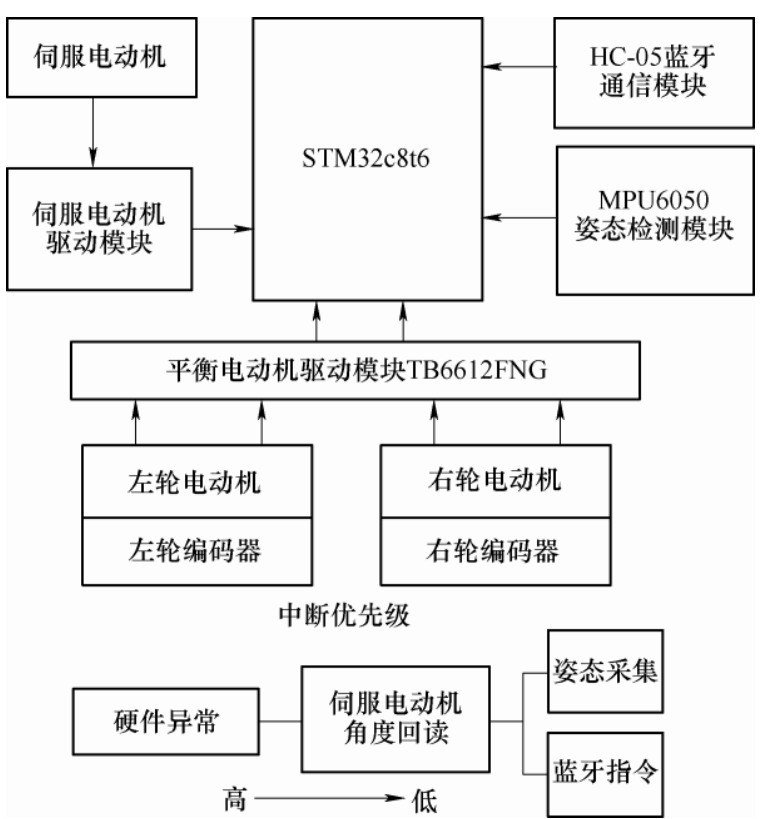

图 21 控制系统结构

跳跃机器人控制系统硬件主要包括主控模块、 驱动模块、通信模块以及传感器模块。主控模块为 STM32c8t6 芯片, 具有体积小、程序存储容量大等 优势。驱动模块包括跳跃、轮式移动的电动机以及 相应的驱动电路。通信模块主体为 HC-05 蓝牙通信 模块, 实现同远程上位机间的通信。传感器模块包 括伺服电动机与平衡电动机的编码器以及姿态检测 的陀螺仪模块。

与主控芯片相匹配的软件系统主要为嵌入式实 时操作系统与基于中断响应的控制策略。为了保证 控制器性能的高效利用对控制器进行嵌入式实时操 作系统 FreeRTOS 的移植。利用中断响应快、控制 性能良好等特点对各部分模块进行中断控制, 具体 的优先级如图 21 所示。

\section{5 仿真与试验}

\section{1 虚拟仿真}

使用 ADAMS 建立如图 22 所示的动力学仿真

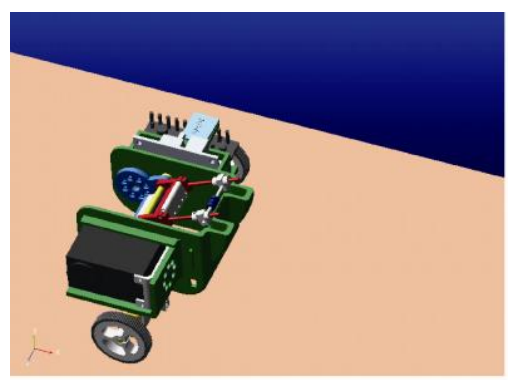

图 22 动力学仿真模型 
模型, 完成跳跃的虚拟仿真, 验证跳跃原理的有效 性以及测试跳跃性能。

通过虚拟仿真可得初始时机器人整体质心在跳 跃过程中的变化, 如图 23 所示。仿真结果表明跳跃 机器人整体在空中做斜抛运动, 可实现高度为 $0.204 \mathrm{~m}$, 距离为 $0.210 \mathrm{~m}$ 的跳跃动作。

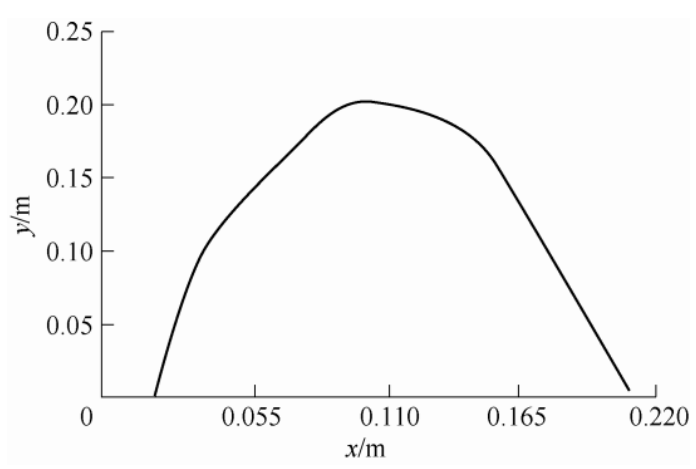

图 23 初始质心变化

\section{2 跳跃试验}

使用 3D 打印技术完成物理样机的制作与封装, 如图 24 所示。机器人整体长 $15 \mathrm{~cm}$ 、宽 $9 \mathrm{~cm}$ 、高 $7 \mathrm{~cm}$, 封装后整体质量 $268.6 \mathrm{~g}$ 。

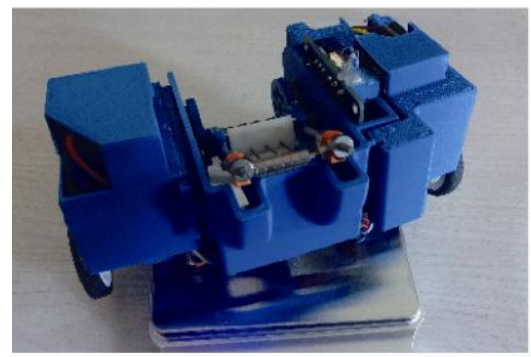

(a) 整体结构

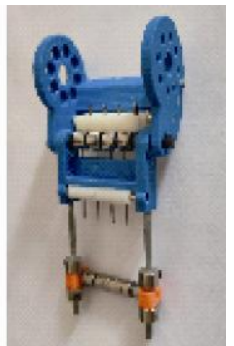

(b) 跳跃结构
图 24 实物模型

跳跃试验测试结果表明复合结构式的跳跃机器 人具备快速运动性能, $1 \mathrm{~s}$ 内完成能量蓄积与释放, 起跳后 $0.3 \mathrm{~s}$ 到达最大高度 $20 \mathrm{~cm}$ 处, 跳跃时间持 续 $0.5 \mathrm{~s}$, 跳跃距离 $15 \mathrm{~cm}$, 如图 25 所示。通过与

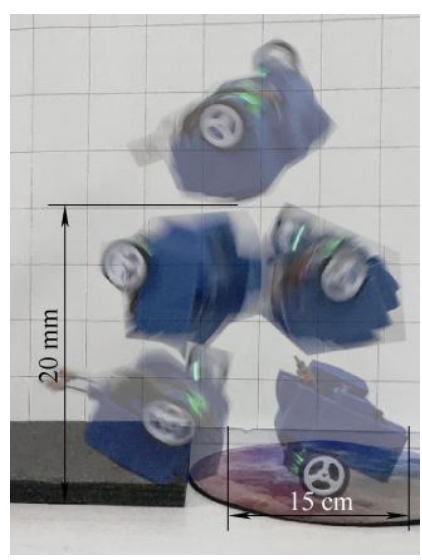

图 25 跳跃测试
ADAMS 虚拟样机仿真对比发现, 机器人在跳跃高 度上与仿真一致, 但在水平距离上约有 $6 \mathrm{~cm}$ 左右 的误差, 初步分析原因为齿节杆端部与地面有较大 的滑动。

\section{3 轮式移动与复位试验}

复合结构式跳跃机器人可实现平衡轮式移动, 测试结果显示机器人可在 $1 \mathrm{~s}$ 内实现 $50 \mathrm{~cm}$ 的快速 移动, 如图 26 所示。

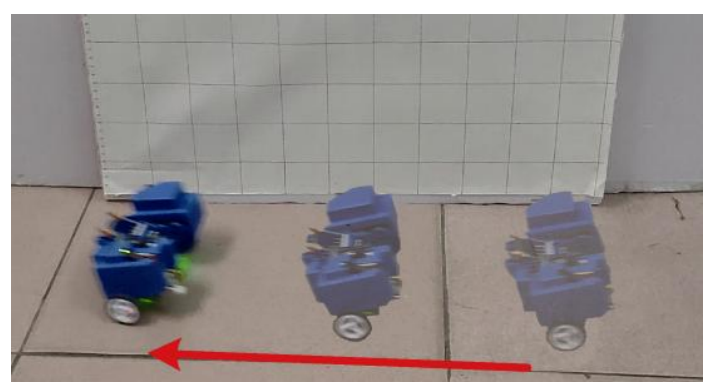

图 26 自平衡移动

跳跃机器人在跳跃后经常性的发生姿态的翻 转, 这对下一次的跳跃造成的影响较大, 因此自主 的姿态复位对跳跃机器人快速连续性运动具有重大 意义。如图 27 所示, 复合结构式跳跃机器人在跳跃 后若发生翻转可实现在 $0.5 \mathrm{~s}$ 内实现姿态复位, 试 验原理与图 5 一致。

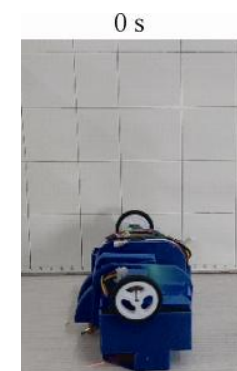

(a) 翻转姿态

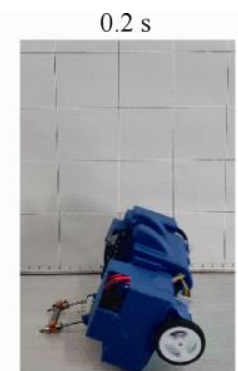

(b) 复位姿态

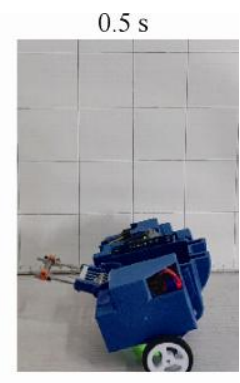

(c) 姿态转正
图 27 姿态复位

\section{6 结论}

（1）基于形态功能学对弹尾虫运动结构及跳跃 运动机制进行分析, 结合平面连杆机构与变胞运动 副的优势实现跳跃原理结构的设计。使用拉格朗日 法完成跳跃原理机构动力学正问题的建模, 得出机 构跳跃的具体过程以及杆件姿态, 同时对最终的结 果进行了数值验证。

（2）针对弹尾虫跳跃结构的兼容性, 对跳跃原 理机构进行了平衡轮式结构的复合设计, 并搭建了 机器人的软硬件控制系统。复合结构式的设计使得 机器人在具备跳跃越障能力的基础上拥有高效的轮 
式移动能力, 同时基于嵌入式实时操作系统 FreeRTOS 与中断响应策略为机器人运动与传感、指 令控制、信息反馈等功能提供了方便。

(3) 通过 ADAMS 建立了虚拟样机对跳跃原理 及跳跃性能进行理论测试。使用 3D 打印技术完成 物理样机的制作。通过试验测试表明此机器人具备 快速连续运动性能, $1 \mathrm{~s}$ 内完成能量蓄积与释放, $0.5 \mathrm{~s}$ 内实现姿态复位; 具备多运动模式, 可实现高 $20 \mathrm{~cm}$ 、远 $15 \mathrm{~cm}$ 的跳跃动作, 之后可完成跳跃后 的姿态复位, 平衡轮式移动, 以及传统平衡小车难 以实现的倒地后自主平衡复位。

后续研究内容主要包括：跳跃结构优化; 齿节 杆末端与地面受力特性分析; 针对弹尾虫水路两栖 的跳跃能力, 对跳跃原理机构进行水面跳跃测试与 分析等。

\section{参 考 文 献}

[1] 莫小娟, 葛文杰, 赵东来, 等. 微小型跳跃机器人研究 现状综述[J]. 机械工程学报, 2019, 55(15): 109-123. MO Xiaojuan, GE Wenjie, ZHAO Donglai, et al. Review: Research status of miniature jumping robot[J]. Journal of Mechanical Engineering, 2019， 55(15): 109-123.

[2] ZAITSEV V, GVIRSMAN O, BEN H U, et al. A locust-inspired miniature jumping $\operatorname{robot}[\mathrm{J}]$. Bioinspir Biomim, 2015, 10(6): 066012.

[3] JUNG G P, CHO K J. Froghopper-inspired direction changing concept for miniature jumping robots[J]. Bioinspir Biomim, 2016, 11(5): 056015.

[4] HALDANE D W, PLECNIK M M, YIM J K, et al. Robotic vertical jumping agility via series-elastic power modulation[J]. Science Robotics, 2016， 1(1): eaag2048.

[5] LI S, RUS D. JelloCube: A continuously jumping robot with soft body[J]. IEEE/ASME Transactions on Mechatronics, 2019, 24(2): 447-458.

[6] 陈殿生, 郑万军, 黄宇, 等. 弹跳机器人翻转机构的设 计与优化[J]. 机械工程学报, 2011, 47(1): 17-23. CHEN Diansheng, ZHENG Wanjun, HUANG Yu, et al. The design and optimization of a hopping robot's tipping mechanism[J]. Journal of Mechanical Engineering, 2011, 47(1): $17-23$.

[7] ZHANG J, SONG G, LI Y, et al. A bio-inspired jumping robot: Modeling, simulation, design, and experimental results[J]. Mechatronics, 2013, 23(8): 1123-1140.

[8] 张小飞, 张延恒, 孙汉旭, 等. 可跳跃式两轮机器人的
设计与动力学分析 $[\mathrm{J}]$. 机器人, 2014, 36(3): 355-361. ZHANG Xiaofei, ZHANG Yanheng, SUN Hanxu, et al. Design and dynamics analysis of a two-wheel robot with hopping ability[J]. Robot, 2014, 36(3): 355-361.

[9] 鲍延年, 蒋海义, 罗天洪, 等. 折叠翼弹跳机器人机构 设计及运动性能分析[J]. 机器人, 2019, 41(2): 175-184. BAO Yannian, JIANG Haiyi, LUO Tianhong, et al. Mechanism design and locomotion performance analysis of the foldable-wing jumping robot[J]. Robot, 2019, 41(2): 175-184.

[10] 毕役. 新发现的弹尾虫[J]. 百科知识, 2013(19): 31 . BI Yi. Newly discovered bullettail[J]. Encyclopedic Knowledge, 2013(19): 31.

[11] SUDO S, SHIONO M, KAINUMA T, et al. Observations on the springtail leaping organ and jumping mechanism worked by a spring[J]. Journal of Aero Aqua Biomechanisms, 2013, 3(1): 92-96.

[12] SUDO S, KAINUMA T, YANO T, et al. Jumps of water springtail and morphology of the jumping organ[J]. Journal of the Japanese Society for Experimental Mechanics, 2015, 15: s117-s124.

[13] 张军. 仿蝗虫弹跳机器人运动机理与控制方法研究 $[D]$. 南京: 东南大学, 2013.

ZHANG Jun. Research on motion mechanism and control method of locust-like bounce robot[D]. Nanjing : Southeast University, 2013.

[14] YU D, DING Y, MA Y. Revision of Tomocerus similis Chen \& Ma, with discussion of the kinoshitai complex and the distal tibiotarsal chaetae in Tomocerinae (Collembola, Tomoceridae) [J]. Zootaxa, 2017, 4268(3): 395-410.

[15] ZHAO J, XU J, GAO B, et al. MSU jumper: A singlemotor-actuated miniature steerable jumping $\operatorname{robot}[\mathrm{J}]$. IEEE Transactions on Robotics， 2013， 29(3): 602-614.

作者简介: 陈子明, 男, 1984 年出生, 博士, 副教授。主要研究方向为 空间少自由度并联机构的设计和分析理论。

E-mail: chenzm@ysu.edu.cn

卢杰, 男, 1994 年出生, 硕士研究生。主要研究方向为跳跃机器人。 E-mail: lujie1994@foxmail.com

邓朋, 男, 1993 年出生, 硕士研究生。主要研究方向为并联机器人的标 定技术研究。

E-mail: dengp0927@foxmail.com

郭玉, 男, 1995 年出生, 硕士研究生。主要研究方向为机器人运动控制。 E-mail: gajade@foxmail.com

李绝文(通信作者), 女, 1966 年出生, 博士, 教授。主要研究方向为机 器人技术及应用。

E-mail: ywl@ysu.edu.cn 